\title{
Tratamiento de un aneurisma serpentino gigante de arteria cerebral media con bypass de alto flujo desde la arteria carótida interna petrosa
}

\author{
J.M. González-Darder; V. Quilis-Quesada; P. González-López; L. Real-Peña y V. Cortés-Doñate*
}

Servicios de Neurocirugía y de *Neurofisiología. Hospital Clínico Universitario. Valencia.

\section{Resumen}

Se presenta un caso de aneurisma gigante del segmento M1 de la arteria cerebral media (ACM), de tipo serpentino, tratado mediante bypass de alto flujo con vena safena externa desde la arteria carótida interna petrosa. Se describen los pasos de la cirugía y se destacan las dificultades de la técnica. Se discuten los elementos a considerar en la toma de decisiones para la indicación, diseño y realización del bypass en el tratamiento de los aneurismas de la ACM.

PALABRAS CLAVE. Aneurisma cerebral gigante. Aneurisma serpentino, Arteria carótida interna petrosa. Bypass intra-intracraneal.

Treatment of a giant serpentine type middle cerebral artery aneurysm with a high-flow bypass from the petrous internal carotid artery

\section{Summary}

A case report of a giant serpentine type aneurysm arising from the M1 segment of the middle cerebral artery (MCA) treated with a high-flow external saphenous vein graft from the petrous segment of the internal carotid artery is presented. The steps and challenges of this demanding surgical technique are also described. The elements to be taken into consideration in the indication, design and realization of the bypass surgery in the treatment of the MCA aneurysms are discused.

KEY WORDS. Giant cerebral aneurysm. Serpentine aneurysm. Petrous internal carotid artery. Intracranial-intracranial bypass

\section{Introducción}

El bypass es una técnica alternativa a la exclusión

Recibido: 31-01-11. Aceptado: 4-04-11 directa con clipaje microquirúrgico de los aneurismas cerebrales gigantes $1,3,4,6,8,10,13$. En el presente trabajo describimos el tratamiento de un aneurisma gigante del segmento M1 de la arteria cerebral media (ACM), de tipo serpentino, mediante bypass de alto flujo con vena safena externa desde la arteria carótida interna (ACI) petrosa al ramo superior postbifurcación de la ACM, con posterior exclusión quirúrgica del aneurisma.

\section{Caso clínico}

Mujer de 35 años de edad, sin antecedentes de interés, con clínica progresiva de varios meses de evolución de cefalea, bradipsiquia y torpeza en hemicuerpo izquierdo. En la exploración neurológica destaca paresia facial inferior izquierda y hemiparesia $4+/ 5$ izquierda, de predominio braquial. Los estudios de imagen (Fig. 1 A-E) mostraban una masa silviana derecha de $55 \times 39 \times 33 \mathrm{~mm}$ con signos de flujo intralesional y edema perilesional, que producía compresión del pedúnculo cerebral y desplazamiento de línea media, sugestiva de aneurisma gigante de la ACM con trombosis intraluminal parcial. La angiografía cerebral confirmó que se trataba de una lesión aneurismática del segmento M1 de la ACM derecha, con luz vascular de trayecto tortuoso y de flujo lento, gran dilatación inicial y otra más pequeña distal, asentada en le segmento M1 de la ACM inmediatamente antes de su bifurcación. Un estudio de angio-TAC con reconstrucción 3D mostraba un segmento M1 de la ACM previo al aneurisma de $1 \mathrm{~cm}$ y que la bifurcación de la $\mathrm{ACM}$ se producía prácticamente al final del aneurisma.

La paciente fue intervenida bajo anestesia general, monitorización neurofisiológica de potenciales evocados somatosensoriales (PESS) por estimulación de los nervios mediano y tibial posterior y potenciales evocados motores (PEM) por estimulación eléctrica cortical transcraneal (Nihon Koden Neuropack) y control de flujo con sonda microdoppler (Microvascular $20 \mathrm{MHZ}$ Intraoperative Doppler System, Vascular Technology VTI). Se realizó un abordaje frontotemporal transcigomático derecho, con apertura de las cisternas para favorecer la relajación cerebral y 


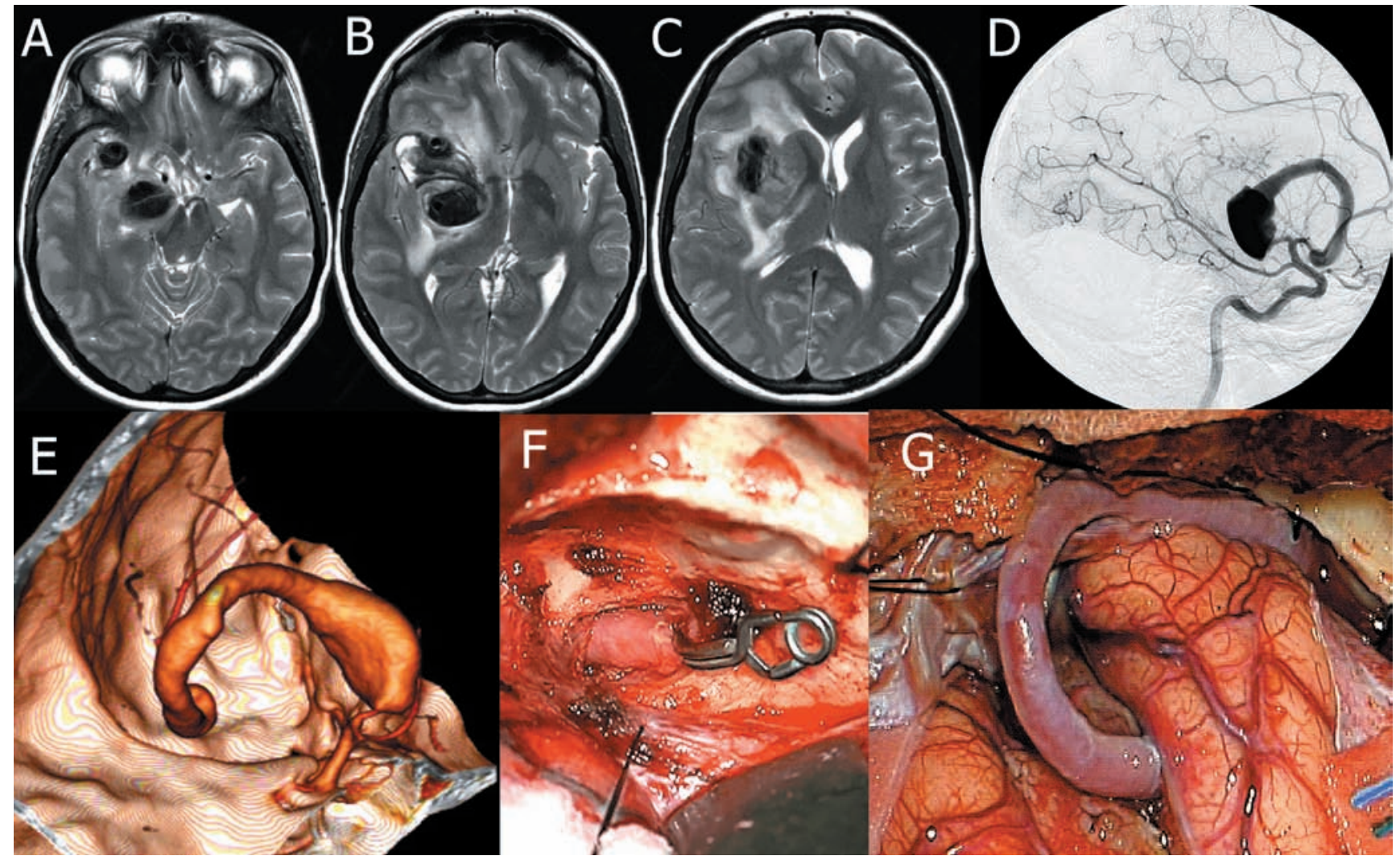

Figura 1. Imágenes de RNM potenciadas en T2 del aneurisma, que muestran trombosis parcial y un canal de flujo intralesional, edema perilesional y efecto de masa $(A, B, C)$. La angiografía lateral muestra el canal de flujo en el segmento M1 de la ACM, con una gran dilatación inicial y otra menor distal (D). El estudio de angio-TAC-3D muestra un corto segmento preaneurismático y un insignificante segmento postaneurismático prebifurcación de la ACM (E). Imágenes intraoperatorias del segmento de ACI petrosa expuesto con el clip temporal proximal $(F)$ y el bypass completado y funcionando $(G)$.

explorar el aneurisma, seguido del abordaje extradural a la fosa temporal para exponer la ACI petrosa en una extensión de $14 \mathrm{~mm}$, desde el borde posterior de la tercera rama del trigémino (V3) hasta el ganglio geniculado (Fig. $1 F)$. Se realizó una clinoidectomía anterior intradural parcial. Tras tomar un injerto de safena externa de la pierna derecha y proceder a la anticoagulación con 5.000 $\mathrm{u}$ de heparina, se colocaron clips temporales en la ACI petrosa proximal, arteria oftálmica y ACI supraclinoidea y se procedió a la sutura terminolateral de la safena a la ACI petrosa con puntos sueltos de $8 / 0$. Durante la sutura se apreciaba un pequeño flujo retrógrado, atribuido a las ramas cavernosas de la ACI. Se retiraron los clips temporales y, comprobada la estanquidad de la sutura, se colocaron clips temporales en el ramo superior postbifurcación de la ACM, realizando la sutura terminolateral de la safena a la misma con puntos sueltos de 9/0 (Fig. 1F). Asegurada la estanquidad de esta nueva anastomosis y el flujo por el bypass, se procedió a la exclusión del aneurisma mediante la colocación de sendos clips en el segmento preaneurismático y en el postaneurismático de la ACM, este último un miniclip, dado el mínimo segmento de ACM disponible entre el aneurisma y la bifurcación. Debido al tamaño de la lesión y a la disposición de la misma, se decidió no extirparla, procediendo al cierre de la craneotomía. Durante la cirugía se mantuvo normotensión, se administró manitol y nimodipino y no se emplearon relajantes musculares. La monitorización neurofisiológica no detectó en ningún momento caída de amplitudes o aumento de latencias en los PESS ni PEM. El control con microdoppler mostró buena velocidad de flujo tras las diferentes maniobras vasculares realizadas y al final de la cirugía el bypass aparecía lleno y pulsátil. Tras la cirugía se colocó un sensor de presión intracraneal (PIC)(CAMINO, Integra) y la paciente fue trasladada a la Unidad de Reanimación, iniciando tratamiento antiagregante con ácido acetilsalicílico a bajas dosis. Se apreció en la TAC de control precoz un discreto aumento de volumen del aneurisma por la trombosis intraluminal, pero los valores de PIC se mantuvieron por debajo de 


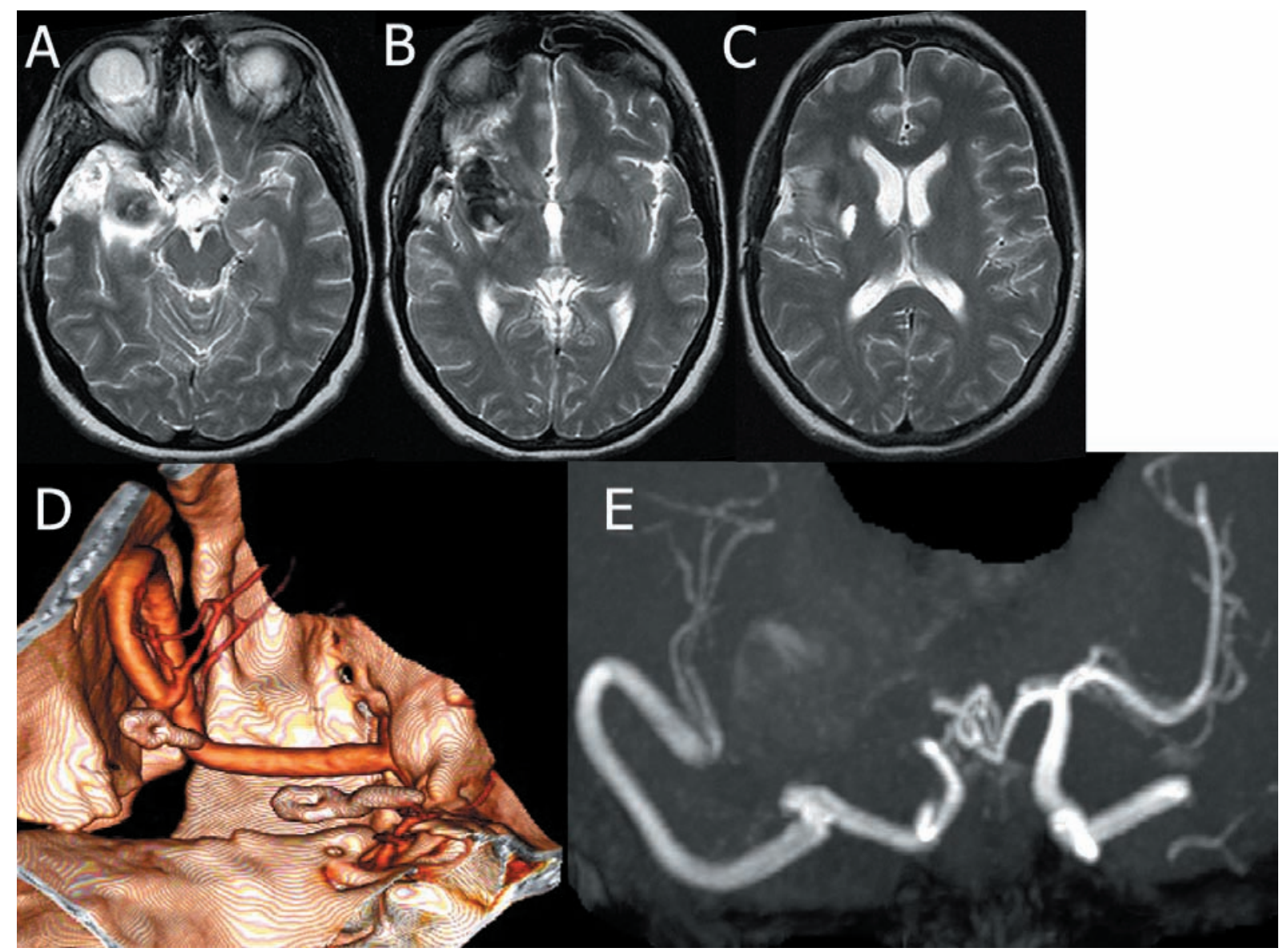

Figura 2. Imágenes de RNM potenciadas en T2 del aneurisma tras la cirugía, que muestran la ausencia de edema perilesional, retracción de la lesión y ausencia de efecto de masa $(A, B, C)$. En estudio de angio-TAC-3D $(G)$ y RNM-vascular (H) postoperatorio demuestra la exclusión del aneurisma, flujo por el polígono de Willis, bypass y ambos troncos postbifurcación de la ACM, apreciándose los clips proximal y distal colocados en la ACM.

20mmHg. Al alta hospitalaria no había déficits y se mantuvo el tratamiento antiagregante. A los seis meses, un angio-TAC con reconstrucción 3D y un estudio de RNM vascular muestran la permeabilidad del bypass, el flujo por el polígono de Willis a través de la ACI y el flujo distal en la ACM a través del bypass, mientras que en la RNM se aprecia la desaparición del efecto de masa de la lesión y del edema perilesional (Fig. 2).

\section{Discusión}

Algunos aneurismas cerebrales gigantes sólo pueden ser tratados mediante bypass, seguido o no de la exclusión y de la exéresis quirúrgica del aneurisma ${ }^{1,3,4,6,8,10,13}$. Las características del aneurisma gigante tratado se corresponden con las de los aneurismas serpentinos, término descriptivo que sirve para designar a los aneurismas trombosados que poseen un canal vascular tortuoso no endotelizado en su seno que conecta la entrada y salida del vaso a través de la lesión. Este término fue introducido por Segal y McLaurin ${ }^{11}$ al describir dos casos de este raro subtipo de aneurismas gigantes ${ }^{2,3}$. El aneurisma serpentino se formaría tras la trombosis intraluminal total de un aneurisma gigante, que sería perforado posteriormente por el efecto 'jet' de la corriente sanguínea. Una hipótesis alternativa sería la trombosis intraluminal de un aneurisma sacular gigante por el llamado efecto Coanda, donde el efecto 'jet' de la sangre entrando en el aneurisma hace que el flujo se dirija hacia la pared contralateral, dejando áreas de flujo turbulento más lento donde se produciría la trombosis intraluminal. Se describen especialmente en la ACM y se trata de lesiones sintomáticas y evolutivas por lo que requieren frecuentemente tratamiento.

Para el diseño del tratamiento quirúrgico de la lesión descrita se tomaron en consideración los siguientes factores: características del aneurisma, opciones de reparación directa, tipo de bypass y, finalmente, riesgo del paciente de sufrir eventos isquémicos durante la cirugía. La ausen- 
cia de cuello, la larga distancia entre el punto de entrada y salida de la ACM en el aneurisma junto al volumen de la lesión y el desconocimiento del nivel de organización del trombo intraluminal imposibilitaban cualquier intento razonable de exclusión por clipaje o de reconstrucción de la luz vascular tras aneurismorrafia. La reconstrucción directa de la ACM con injerto terminoterminal tras la resección del aneurisma se consideró inapropiada por la profundidad del cabo proximal, falta de un segmento adecuado distal prebifurcación y el largo periodo de isquemia necesario en todo el territorio de la ACM, por lo que se decidió el bypass. Actualmente se clasifican los bypass cerebrales dependiendo del flujo que son capaces de vehicular ${ }^{6}$. Los bypass de alto flujo aportan entre 100 y $200 \mathrm{ml} / \mathrm{min}$ y se hacen con la vena safena externa, precisándose en este caso vasos receptores de tamaño adecuado para acoger y distribuir dicho flujo. En nuestro caso, el vaso receptor tenía que ser la propia bifurcación de la ACM o uno de los ramos postbifurcación, con lo que aseguraría el flujo distal completo si se podía colocar un clip antes de la bifurcación o, en caso contrario, mediante la técnica de la 'doble anastomosis's . El vaso dador usado habitualmente en los bypass de alto flujo con safena es la arteria carótida externa (ACE) o una de sus ramas (bypass extra-intracraneal) ${ }^{1,11}$. Sin embargo, este es un bypass extra-anatómico, con una serie de problemas relacionados: gran longitud, mayor número de válvulas, peligro de compresión externa o torsión por movimientos que, entre otros, condicionan una cierta tasa de fracasos precoces y tardíos ${ }^{4,6,13}$. Frente a ello, el bypass desde la propia ACI (bypass intra-intracraneal) tendría ventajas, incluyendo una distribución del flujo más fisiológica y la conservación anatómica de la $\mathrm{ACE}^{10}$. En nuestro caso, el vaso dador elegido fue la ACI petrosa. En lo que se refiere al riesgo de isquemia durante la realización de bypass o por la exclusión del aneurisma con el cierre del segmento M1 de la ACM, un elemento impredecible siempre es la existencia y el valor funcional de las ramas perforantes y lenticuloestriadas del segmento M1 donde asentaba el aneurisma ${ }^{9}$ aunque sea una observación clínica habitual que en los aneurismas gigantes que no se encuentran ramas perforantes. Los resultados de los tests de oclusión preoperatorios con balón no son definitivos, no están exentos de complicaciones y en nuestro caso podría seguirse de trombosis de la ACM o embolismo desde el aneurisma. Por todo ello, decidimos actuar de acuerdo a los datos directos obtenidos con la monitorización neurofisiológica intraoperatoria de los PESS y PEM.

El bypass intra-intracraneal desde la ACI petrosa fue publicado por primera vez por Spetzler et al. ${ }^{12}$. Sin embargo, esta estrategia no ha tenido mucho predicamento debido a sus grandes requerimientos técnicos, destacados por todos los autores y relacionados con la dificultad del acceso a la ACI petrosa con exposición limitada y profunda de la misma que dificultan la sutura ${ }^{5,7}$. Sin embargo, varios estudios anatómicos describen de forma detallada el acceso a la ACI petrosa, demostrando que se pueden exponer hasta $17 \mathrm{~mm}$ de la misma $^{5,7}$. El entrenamiento en cadáveres y el acceso quirúrgico repetido a la zona son esenciales para un abordaje quirúrgico seguro, en el que hay que exponer la ACI petrosa desde el ganglio geniculado hasta el borde posterior de $\mathrm{V} 3^{5,7}$. La dificultad de la sutura en una zona profunda se compensa con el grosor de la pared de la ACI, semejante a la de la safena externa y por el buen calibre de ambos vasos. En nuestro caso, el segmento expuesto de la ACI fue de $14 \mathrm{~mm}$, suficiente para la sutura de la safena externa de $5 \mathrm{~mm}$ de diámetro. Nosotros conseguimos un espacio adicional eludiendo el clip distal mediante el cierre temporal distal de la ACI a nivel paraclinoideo. Como contrapartida, esta estrategia supone la realización de maniobras suplementarias, concretamente una clinoidectomía parcial que permite localizar la salida de la arteria oftálmica para el cierre temporal tanto de la ACI supraclinoidea como de la oftálmica. Alternativamente, puede hacerse una clinoidectomía anterior intradural para cierre temporal de la ACI en su segmento clinoideo. Aun así, ninguna de estas dos maniobras evita un pequeño flujo retrógrado hacia la arteriotomía procedente de las ramas intracavernosas de la $\mathrm{ACI}^{7}$.

\section{Conclusiones}

El bypass intra-intracraneal de alto flujo con vena safena externa desde la ACI petrosa es una técnica útil en el tratamiento de los aneurismas cerebrales gigantes de la ACM inabordables de forma directa. La sutura proximal requiere una adecuada exposición de la ACI petrosa y es muy exigente desde un punto de vista técnico. La sutura distal debe hacerse en vasos de calibre adecuado para recibir y distribuir el flujo, concretamente la propia bifurcación o uno de sus ramos de primer orden. El diseño de la estrategia de tratamiento debe ser cuidadoso y basado en una adecuada exposición quirúrgica y dominio de la técnica microquirúrgica. La monitorización neurofisiológica intraoperatoria puede ser de ayuda a la hora de tomar decisiones, en especial, para decidir la exclusión intraoperatoria del aneurisma.

\section{Agradecimientos}

Los autores quieren agradecer la colaboración de los miembros de los Servicios de Cirugía Cardiovascular y de Anestesiología y Reanimación del Hospital Clínico Universitario de Valencia. 


\section{Bibliografía}

1. Bisson, E.F., Visioni, A.J., Tranmer, B., Horgan, M.A.: External carotid artery to middle cerebral artery bypass with the saphenous vein graft. Neurosurgery 2008; 62(ONS Suppl 1): ONS134-ONS139.

2. Christiano, L.D., Gupta, G., Giacomo, C.J.P., Gandhi, C.D.: Giant serpentine aneurysms. Neurosurg Focus 2009; 26: E5.

3. González-Darder, J.M., Pesudo-Martínez, J.V., Wiederman, O.: Aneurismas cerebrales gigantes. Rev Neurol 2006; 43: 357-365.

4. Lawton, M.T., Quiñones-Hinojosa, A.: Double reimplantation technique to reconstruct arterial bifurcations with giant aneurysms. Neurosurgery 2006; 58(ONS Suppl 4): ONS-347-ONS-354.

5. Liu, J.K., Fukushima, T., Sameshima, T., Al-Mefty, O., Couldwell, W.T.: Increasing exposure of the petrous internal carotid artery for revascularization using the transzygomatic extended middle fossa approach: a cadaveric morphometric study. Neurosurgery 2006; 59 (ONS Suppl 4): ONS309ONS319.

6. Mohit, A.A., Sekhar, L.N., Natarajan, S.K., Britz, G.W., Ghodke, B.: High-flow bypass grafts in the management of complex intracranial aneurysms. Neurosurgery 2007; 60 (ONS Suppl): ONS105-ONS123..

7. Osawa, S., Rhoton, A.L., Tanriover, N., Shimizu, S., Fujii, K.: Microsurgical anatomy and surgical exposure of the petrous segment of the internal carotid artery. Neurosurgery 2008; 63 (ONS Suppl 2): ONS210-ONS239.

8. Roda, J.M., González-Llanos, F., Pascual, J.M.: Papel de la anastomosis extra-intracraneal y de la terapia endovascular intervencionista en el tratamiento de los aneurismas complejos cerebrales. Neurocirugía 2002; 13: 365-370.

9. Rhoton, A.L.: The supratentorial arteries. Neurosurgery 2002; 51 (Suppl 1): 53-120.

10. Sanai, N., Zador, Z., Lawton, M.T.: Bypass surgery for complex brain aneurysms: an assesment of intracranial-intracranial bypass. Neurosurgery 2009; 65: 670-683.

11. Segal HD, McLaurin RL: Giant serpentine aneurysm. Report of two cases. J Neurosurg 1977; 46: 115-120.

12. Spetzler, R.F., Fukushima, T., Martin, N., Zabramski, J.M.: Petrous-carotid to intradural carotid saphenous vein graft for intracavernous giant aneurysm, tumor, and occlusive cerebrovascular disease. J Neurosurg 1990; 73: 496501.

13. van Doormaal, T.P.C., van der Zwan, A., Verweij, B.H., Han, K.S., Langer, D.J., Tulleken, C.A.F: Treatment of giant middle cerebral artery aneurysms with a flow replacement bypass using the excimer laser-assisted nonocclusive anastomosis technique. Neurosurgery 2008; 63: 12-22.

González-Darder, J.M.; Quilis-Quesada, V.; GonzálezLópez, P.; Real-Peña, L.; Cortés-Doñate, V.: Tratamiento de un aneurisma serpentino gigante de arteria cerebral media con bypass de alto flujo desde la arteria carótida interna petrosa. Neurocirugía 2011; 22: 429-433.

Correspondencia: Dr. José M González Darder. Servicio de Neurocirugía. Hospital Clínico Universitario. Avda. Blasco Ibáñez, 17. 46010-Valencia

gonzalez_jos@gva.es 